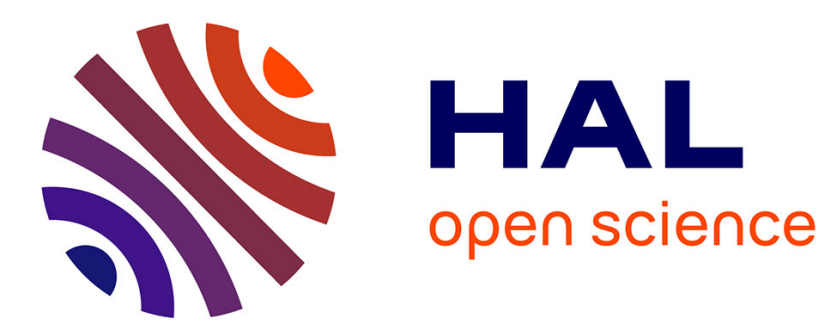

\title{
A standardization method to compare isotonic vs. isokinetic eccentric exercises
}

Gaël Guilhem, Arnaud Guével, Christophe Cornu

\section{To cite this version:}

Gaël Guilhem, Arnaud Guével, Christophe Cornu. A standardization method to compare isotonic vs. isokinetic eccentric exercises. Journal of Electromyography and Kinesiology, 2010, 20, pp.1000 - 1006. 10.1016/j.jelekin.2010.03.009 . hal-01565495

\section{HAL Id: hal-01565495 https://hal-insep.archives-ouvertes.fr/hal-01565495}

Submitted on 19 Jul 2017

HAL is a multi-disciplinary open access archive for the deposit and dissemination of scientific research documents, whether they are published or not. The documents may come from teaching and research institutions in France or abroad, or from public or private research centers.
L'archive ouverte pluridisciplinaire HAL, est destinée au dépôt et à la diffusion de documents scientifiques de niveau recherche, publiés ou non, émanant des établissements d'enseignement et de recherche français ou étrangers, des laboratoires publics ou privés. 


\title{
A standardization method to compare isotonic vs. isokinetic eccentric exercises
}

\author{
Gaël Guilhem ${ }^{\mathrm{a}, \mathrm{b}}$, Arnaud Guével ${ }^{\mathrm{a}, *}$, Christophe Cornu ${ }^{\mathrm{a}}$ \\ ${ }^{a}$ University of Nantes, Faculty of Sport Sciences, "Motricité, Interactions, Performance" Laboratory, EA 4334, Nantes, France \\ ${ }^{\mathrm{b}}$ National Institute for Sports (INSEP), Research Department, Laboratory of Biomechanics and Physiology, Paris, France
}

Keywords:

Lengthening contraction

Work

Angular velocity

Surface electromyography

Knee extensors a b s t r a c t

The present study aimed to standardize isotonic (IT) and isokinetic (IK) eccentric exercises by equalizing the amount of work and the angular velocity at two intensity levels, to be able to compare specific effects of these exercise modes on the neuromuscular system. Fourteen subjects participated in three test sessions consisting of two IT and two IK sets on a customized isokinetic dynamometer. IT sets were comprised of 8 eccentric contractions of the knee extensors at $120 \%$ of the maximal repetition (1RM) in the first two sessions, and $100 \%$ in the third session. IK sets were performed at the same mean angular IT velocity and stopped when the amount of work performed corresponded to the IT set. External work, angular velocity and Root Mean Square (RMS) of electromyographic activity of three superficial muscles of the quadriceps femoris were calculated. Results showed concordance of work and angular velocity for each test session. Both modes involved the same number of repetitions at $120 \%$ and fewer repetitions in IK mode at $100 \%$ of $1 \mathrm{RM}$. Work and RMS values remained steady in all sets. This study allowed the standardization of isotonic and isokinetic eccentric exercises, a first step before determining their specific effects on neuromuscular function.

\section{Introduction}

When muscles support a load torque that exceeds the muscular torque, an eccentric contraction is performed. The production of muscular force is associated with an active lengthening. This stretch of the elastic components of the muscle-tendon unit induces the production of high levels of torque during eccentric exercises. Such exercises are commonly used in training programs (Roig et al., 2009) and rehabilitation protocols (Rees et al., 2009). Numerous studies have focused on strength gains and physiological adaptations (e.g. muscular hypertrophy, changes in muscle architecture, rise in neural activation) induced by eccentric training (Blazevich et al., 2007; Guilhem et al., in press-a,b; Hortobagyi et al., 1996a,b; Housh et al., 1998; Komi and Buskirk, 1972; Roig et al., 2009). Eccentric loading has also been suggested to be an effective treatment protocol in the management of chronic tendinopathy, particularly of the Achilles and patellar tendons (Croisier et al., 2007; Kingma et al., 2007; Rees et al., 2009; Stanish et al., 1986). Although most of the studies found that eccentric exercises improve knee function (i.e. ability to undertake physical activity as often quantified by the VISA Score (Visentini et al.,

* Corresponding author. Address: University of Nantes, Faculty of Sport Sciences, "Motricité, Interactions, Performance" Laboratory, EA 4334, 25 bis Bd Guy Mollet 2013, BP 72206, Nantes, F-44000, France. Tel.: +33 (0) 2518372 24; fax: +33 (0) 2 51837210.

E-mail address: arnaud.guevel@univ-nantes.fr (A. Guével).
1998)) and pain, the ability to recommend a specific protocol is limited due to the lack of comparative and controlled studies (Visnes and Bahr, 2007).

Eccentric exercises can be performed against a constant load (isotonic, IT) using body weight (i.e. body weight squat, squat on a decline board), dumbbells or weight lifting bars. During such tasks the device provides a constant resistance throughout the range of the muscle contraction, thus loading the muscles more strongly at the weakest joint angles. Although isoinertial or isoload actions more accurately reflect weight lifting actions (Abernethy et al., 1995; Caruso et al., 2005), this type of task is still often considered an isotonic movement in the literature. Eccentric exercises can also be performed at a constant velocity on an isokinetic (IK) dynamometer. In an IK contraction the velocity remains constant regardless of the resistance performed by the subjects. Consequently, the IK mode is hypothesized to maximally load the muscles through the entire range of motion. These differences in mechanical characteristics may induce different responses of the neuromuscular system. IT (Duclay et al., 2008; Housh et al., 1998) and IK (Hortobagyi et al., 1996a,b; Komi and Buskirk, 1972) eccentric trainings are recognized to improve muscle strength and increase muscle volume and activity. Nevertheless, a comparison between IT and IK eccentric modes in standardized protocol has not been performed. This lack of research may originate from the difficulty in designing protocols that allow for standardization of IT and IK eccentric exercises (Guilhem et al., in press-a,b). 

Previous studies comparing IT and IK concentric exercises have contradicting results regarding which method is most effective at increasing muscular strength (Kovaleski et al., 1995; Smith and Melton, 1981). The apparent discrepancies originate from the protocols set up to compare these two training modes. Authors interested in such a comparison during concentric exercise have discussed several key points. For instance, Smith and Melton (1981) suggested that equalization of the external amount of work performed was a critical question (Smith and Melton, 1981). Moreover, the neuromuscular adaptations induced by eccentric training are highly specific to the angular velocity (Roig et al., 2009). These two parameters were used by recent studies to compare IT and IK standardized concentric exercises. The results showed a different behavior of the neural drive in response to each mode (Remaud et al., 2009). Results showed that both modes involved the same number of repetitions to perform the same amount of external work when IT exercise was set at $80 \%$ of the maximal repetition (1RM) (Remaud et al., 2005). However, the effect of exercise intensity (i.e. external load commonly calculated as a percentage of $1 R M$ or MVC) on the standardization procedure has not yet been considered. Yet external load affects the external torque produced, the amount of work produced for each repetition and the number of repetitions needed to reach the total external work target. Thus the external load could have an effect on the standardization method by modifying the number of repetitions required for each mode. Recently, we designed a new device to perform IT and IK eccentric exercises on the same ergometer which allows for the acquisition of mechanical parameters (i.e. position, torque, angular velocity) under comparable conditions (Guilhem et al., in press-b). The aim of the present study was to develop a standardization methodology for performing IT and IK eccentric exercises by equalizing the amount of external work and the angular velocity performed during both modes. This method was also tested at two intensities commonly used in eccentric exercise to determine potential effects of exercise intensity on the standardization procedure.

\section{Methods}

\subsection{Subjects}

Fourteen healthy male subjects without any previous history of knee injury volunteered to participate in this study. The mean $( \pm \mathrm{SD})$ age, height and body mass of the subjects were $21.5 \pm 2.7$ years, $179.7 \pm 5.7 \mathrm{~cm}$ and $77.7 \pm 9.4 \mathrm{~kg}$, respectively. Subjects were informed regarding the nature, the aims, the risks and discomfort associated to the study before they gave their written consent to participate. This study was approved by a local ethics committee and conducted according to the Helsinki Declaration (1964 revised in 2001).

\subsection{Dynamometry}

Eccentric sessions were performed on a customized ergometer that was previously validated (Guilhem et al., in press-b). Briefly, a plate-loaded resistance training device was implemented to an isokinetic dynamometer (Biodex System 3 Pro, Shirley, NY, USA) to acquire mechanical parameters on the same ergometer in both modes. In IT mode, a constant load by guided masses linked to the dynamometer attachment by a steel wire was applied to the lever arm (Fig. 1). In these conditions, subjects performed eccentric contractions of the knee extensors against a constant torque applied by the resistance training device through the entire range of motion. In the IK mode, subjects performed eccentric contractions at a constant angular velocity. This device also allowed us to stop the exercise when the predetermined amount of work

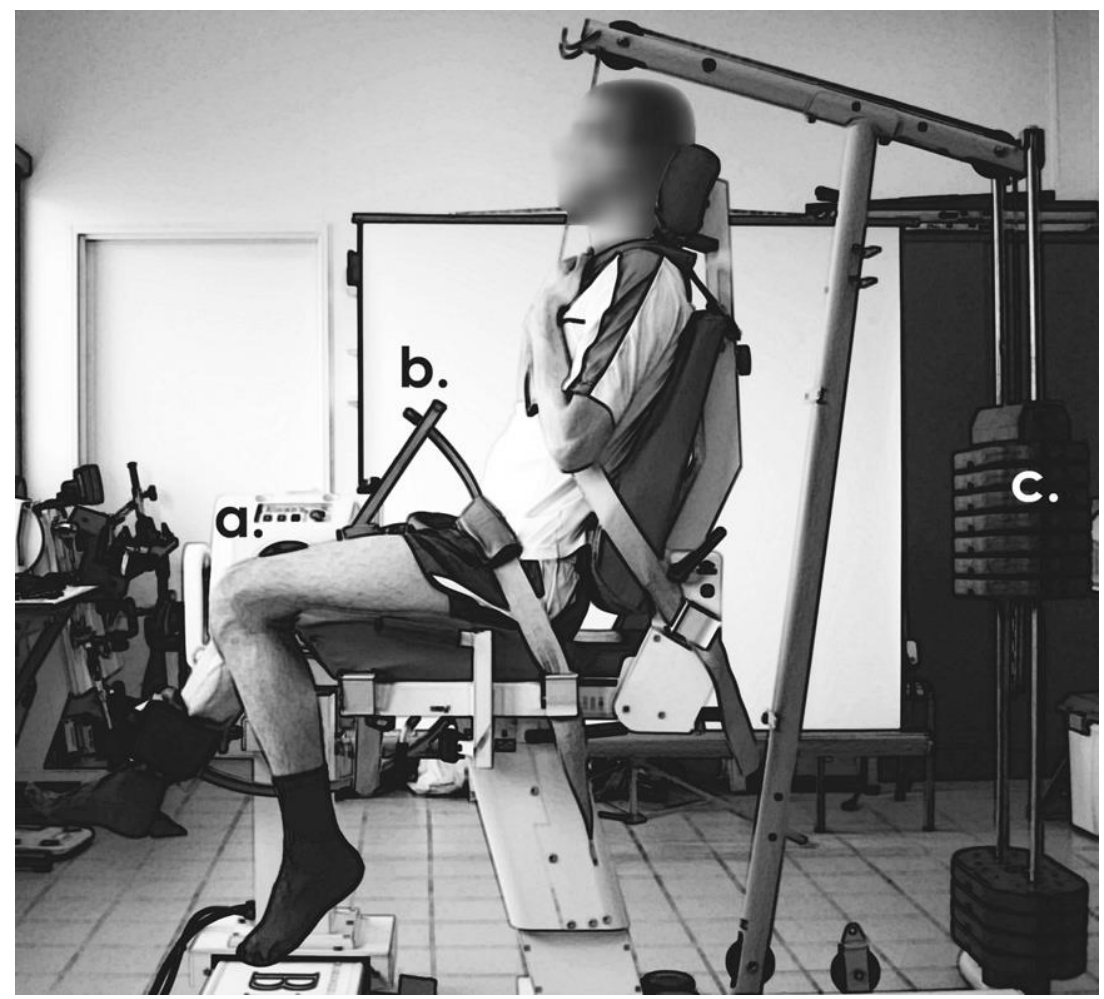

Fig. 1. Picture of the device. A plate-loaded resistance training device was integrated to an isokinetic dynamometer Biodex System 3 (a). Loads (c) were linked to the dynamometer attachment by a wire. The wire passed through two pulleys and followed a half-circle metal piece (b) fixed to the dynamometer attachment. (a) Dynamometer - Biodex System 3 Pro; (b) half-circle metal piece; (c) loads. 
was achieved through the use of visual feedback. Ergometer settings were recorded and reproduced during all sessions. Mechanical signals were recorded at a sampling frequency of $1000 \mathrm{~Hz}$.

\subsection{Electromyography}

Bipolar surface electromyography (EMG) signals were recorded during each test session from surface EMG sensors (DE-2.1, Delsys ${ }^{\circledR}$, Boston, MA, USA) on the vastus lateralis (VL), vastus medialis (VM) and rectus femoris (RF) muscles. Each EMG sensor was made of two parallel silver $(\mathrm{Ag}-\mathrm{AgCl})$ bars with a length of $10 \mathrm{~mm}$. The inter-bar distance was $10 \mathrm{~mm}$. Electrode-skin impedance was reduced using standard skin preparation procedures (Maisetti et al., 2002). According to the SENIAM recommendations (Hermens et al., 2000), surface EMG sensors were placed between the distal tendon and the innervation zone parallel to the direction of the muscle fibers. The positions of the electrodes were labeled with indelible ink during the first test session to ensure the same electrode positioning in each test session. EMG signals were pre-amplified (gain = 10) at the sensor level and sampled at $1000 \mathrm{~Hz}$ via an A/ Dconverter (Bagnoli 16 EMG System, Delsys ${ }^{\circledR}$, Boston, MA, USA; input impedance $>10^{15} \boldsymbol{X}$; common mode-rejection ratio at 60 / $10 \mathrm{~Hz}=92 \mathrm{~dB}$; gain = 1000; bandwidth $=0-400 \mathrm{~Hz}$ ).

\subsection{Experimental design}

Each subject participated in a familiarization session and three test sessions 1 week apart.

\subsubsection{Familiarization session}

The familiarization session allowed the subjects to be familiarized with the customized ergometer and the IT and IK modes. After a 5 min warm-up on a cycloergometer $(100 \mathrm{~W})$ subjects were seated on the ergometer so that the hip was flexed to $85^{\circ}\left(0^{\circ}=\right.$ full hip extension) and began a specific warm-up. After 1 min of rest, subjects performed one maximal isometric voluntary contraction (MVC) in order to determine the first load for the determination of the maximal load the subject could lift in a single concentric contraction (1RM). The first load corresponded to $70 \%$ of the MVC. The load was then progressively increased with a $1 \mathrm{~kg}$ load

\section{Table 1}

Design of the experimental protocol. 1RM: maximal repetition; MVC: maximal isometric voluntary contraction; $\mathrm{IT}_{1}-\mathrm{IT}_{2}$ : first and second isotonic set; $\mathrm{IK}_{1}-\mathrm{IK}_{1}$ : first and second isokinetic set; $\mathrm{X}_{1}-\mathrm{X}_{1}$ : mean of angular velocity in $\mathrm{IT}_{1}$ and $\mathrm{IT}_{2}$; $n_{1}-n_{2}$ : number of repetitions performed in $\mathrm{IK}_{1}$ or $\mathrm{IK}_{2}$ to reach the amount of work performed in $\mathrm{IT}_{1}$ or $\mathrm{IT}_{2}$.

\begin{tabular}{|c|c|c|c|c|}
\hline Order & $\begin{array}{l}\text { Familiarization } \\
\text { session }\end{array}$ & Test session 1 & Test session 2 & Test session 3 \\
\hline 1 & Warm-up & & & \\
\hline 2 & $1 \mathrm{RM}$ & & & \\
\hline 3 & 1 min rest & \multicolumn{2}{|c|}{1 min rest } & 1 min rest \\
\hline 4 & $2 \mathrm{MVC}$ & \multicolumn{2}{|c|}{$\mathrm{IT}_{1}: 8$ repetitions } & $\begin{array}{l}\mathrm{IT}_{1}: 8 \\
\text { repetitions }\end{array}$ \\
\hline & 1 min rest & \multicolumn{2}{|c|}{$120 \% 1 \mathrm{RM}$} & $100 \% 1 \mathrm{RM}$ \\
\hline 5 & $\begin{array}{l}\mathrm{IT}_{1}: 8 \text { repetitions } \\
120 \% 1 \mathrm{RM}\end{array}$ & \multicolumn{2}{|c|}{5 min rest } & 5 min rest \\
\hline 6 & 5 min rest & \multicolumn{2}{|c|}{$\mathrm{IK}_{1}: n_{1}$ repetitions $\mathrm{X}_{1}$} & $\begin{array}{l}\mathrm{IK}_{1}: n_{1} \\
\text { repetitions X } 1\end{array}$ \\
\hline 7 & $\begin{array}{l}2 \mathrm{MVC} \\
1 \text { min rest }\end{array}$ & \multicolumn{2}{|c|}{5 min rest } & 5 min rest \\
\hline 8 & $\begin{array}{l}\mathrm{IK}_{1}: n_{1} \\
\text { repetitions } \mathrm{X}_{1}\end{array}$ & $\begin{array}{l}\mathrm{IT}_{2}: 8 \\
\text { repetitions } \\
120 \% 1 \mathrm{RM}\end{array}$ & $\begin{array}{l}\mathrm{IK}_{2}: n_{1} \\
\text { repetitions } \mathrm{X}_{2}\end{array}$ & $\begin{array}{l}\mathrm{IT}_{2}: 8 \\
\text { repetitions } \\
100 \% 1 \mathrm{RM}\end{array}$ \\
\hline 9 & 1 min rest & 5 min rest & 5 min rest & 5 min rest \\
\hline 10 & 2 MVC & $\begin{array}{l}\mathrm{IK}_{2}: n_{2} \\
\text { repetitions } \mathrm{X}_{2}\end{array}$ & $\begin{array}{l}\mathrm{IT}_{2}: 8 \\
\text { repetitions } \\
120 \% 1 \mathrm{RM}\end{array}$ & $\begin{array}{l}\mathrm{IK}_{2}: n_{2} \\
\text { repetitions X } 2\end{array}$ \\
\hline
\end{tabular}

after a 1 min rest. $1 R M$ was the last load the subjects could lift on the whole range of motion $\left(90-30^{\circ}, 0^{\circ}=\right.$ full knee extension) with a $5^{\circ}$ tolerance. Subjects then completed 10 submaximal eccentric contractions in IT and IK modes before they performed the protocol detailed in Table 1.

\subsubsection{Test sessions}

In each test session, subjects warmed-up and then the 1RM was determined in the same manner as during the familiarization session. In the first (TS1) and second (TS2) test session, subjects performed two IT sets at $120 \%$ of the $1 \mathrm{RM}$ and two IK sets of maximal eccentric contractions of the knee extensors from a knee angle ranging from $30^{\circ}$ to $90^{\circ}$, following the experimental protocol presented in Table 1. In TS2, the second IK set was performed before the second IT set to test the effect of order on the standardization procedure. In the most of training protocols, supra-maximal eccentric sessions are preceded by lower exercise intensity. Numerous studies have shown an effect of exercise intensity (i.e. external load) on muscle activity and consequently on the external torque produced (Babault et al., 2001; Komi et al., 1987; Linnamo et al., 2003). Thus, in order to test the effect of external load on the standardization procedure, the third test session (TS3) consisted in the same protocol as in TS1, but IT sets were performed at $100 \%$ of the $1 \mathrm{RM}$ and IK sets were still performed in maximal conditions.

\subsection{Data analysis}

\subsubsection{Mechanical data}

Torque measurements were gravity and inertia corrected through the overall range of motion (Aagaard et al., 1995; Guilhem et al., in press-b). For each repetition, the amount of external angular work $(W)$ and the mean angular velocity $\partial x \mathrm{P}$ were determined on the whole range of motion (Remaud et al., 2005). W was calculated at each time interval from the muscular torque produced by the subject $\left(T_{\text {mus }}\right)$ and the angular position (h), as expressed by the Eq. (1):

$$
W^{1 / 4}{ }_{\mathrm{h}_{0}}^{\mathrm{Z}_{\mathrm{h}_{n}}} T_{\text {mus }} \cdot d \mathrm{~h}
$$

$\mathrm{W}$ : work (J); $T_{\text {mus }}$ : muscular torque $(\mathrm{N} \mathrm{m}) ; \mathrm{h}$ : angle (rad).

For modes comparison, muscular torque and angular velocity were averaged over $5^{\circ}$ windows.

\subsubsection{Standardization procedure}

IT sets consisted of 8 repetitions at $120 \%$ of $1 \mathrm{RM}$ for the familiarization session, as well as the first and second test sessions. IT mode was set at $100 \%$ of the $1 \mathrm{RM}$ in the third test session. IT angular velocity ranged from 20 to $75^{\circ} \mathrm{s}^{-1}$ across trials. In each IK set, subjects performed $n$ maximal repetitions at a velocity similar to the mean velocity measured during the corresponding isotonic set; where $n$ represented the number of repetitions necessary to reach the amount of work performed in the corresponding isotonic set at the same mean angular velocity (Table 1) (Remaud et al., 2005).

\subsubsection{EMG data}

SEMG data were band-pass filtered using a second order Butterworth filter (bandwidth: $6-400 \mathrm{~Hz}$ ) to remove any motion artefact. The MVC with the highest maximal torque was considered for further analysis. The maximal Root Mean Square (RMS) value was calculated over a time period of $200 \mathrm{~ms}$ (i.e. $100 \mathrm{~ms}$ before and after the time to peak torque) from EMG signals of each muscle (Remaud et al., 2009). For each repetition and for each muscle, a mean RMS value was determined on a time window corresponding to the time required to complete the range of motion comprising the isotonic 
and isokinetic steady states (from $35^{\circ}$ to $80^{\circ}$ ). RMS values of EMG activity of quadriceps femoris were normalized to the maximal RMS value obtained during MVC. RMS values of the three superficial knee extensors were averaged to express a mean knee extensors RMS (Hakkinen et al., 1991).

\subsection{Statistical analysis}

Normality of data was tested using a Kolmogorov-Smirnov test. Two-way ANOVAs (mode $x$ angle) for repeated measures were used to test differences in torque and angular velocity between both exercise modes. The level of concordance for the amount of work and the mean angular velocity between IT and IK modes was assessed using Bland-Altman plots. According to Bland and Altman, the limits of agreement were defined as the mean difference \pm 1.95 SD of the difference, (Bland and Altman, 1986). A Wilcoxon signed rank test was used to analyze potential differences in the number of repetitions between both modes. Trial-to-trial reproducibility of work was determined during the first set of TS2 by calculating the intraclass correlation coefficient (ICC), the standard error of measurement (SEM) and the coefficient of variation (CV). One-way ANOVA for repeated measures was used to test effect of eccentric sets on MVC peak torque. Three-way ANOVAs (mode $\mathrm{X}$ set $\mathrm{X}$ repetitions) for repeated measures were used to test potential differences in work, angular velocity and muscular activity level between repetitions in each set for both mode. If the sphe ricity assumption in repeated measures ANOVAs was violated (Mauchly's test), then a Geisser-Greenhouse correction was used. Post-hoc tests were performed by means of Newman-Keuls proce-

\section{A}
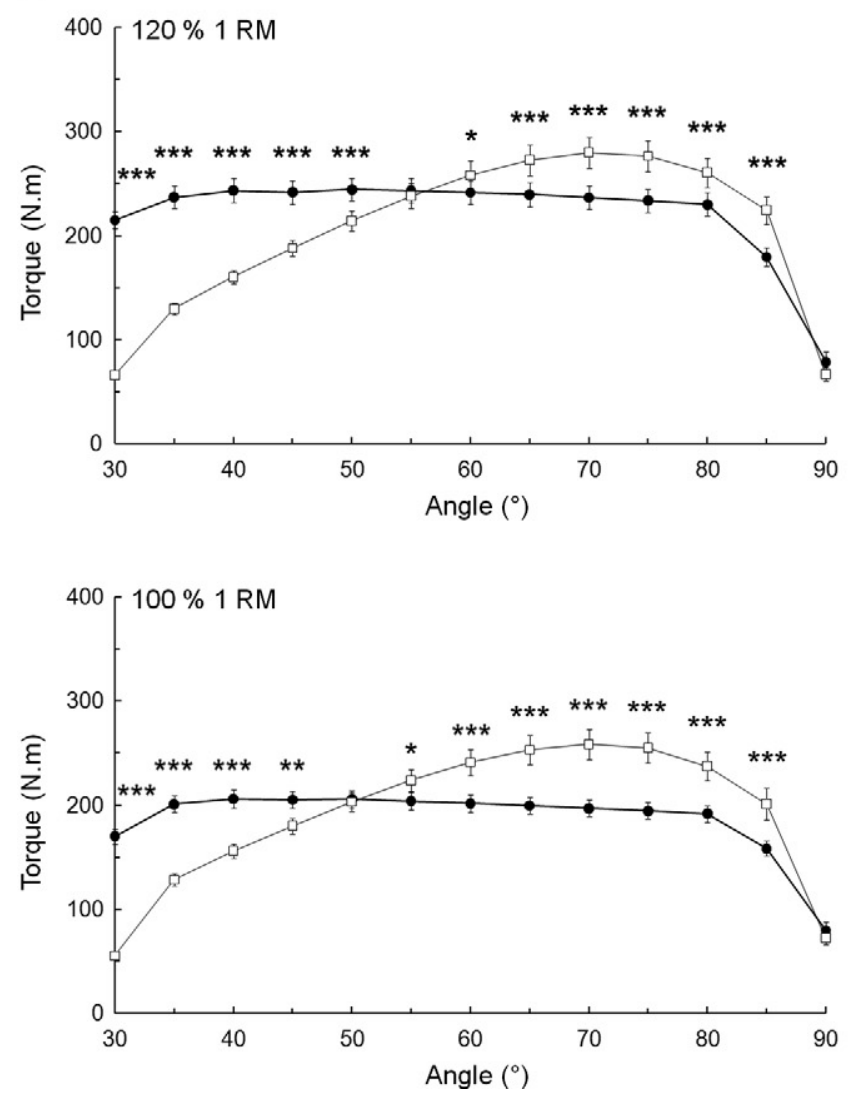

dures. For all tests, the critical level of significance was set at $p<0.05$. Results are presented as mean $\pm \mathrm{SE}$.

\section{Results}

\subsection{Comparison of IT and IK eccentric exercises}

The two-way ANOVAs revealed a mode $x$ angle interaction effect for the torque-angle $(p<0.0001)$ and velocity-angle $(p<0.0001)$ relationships at all test sessions. Post-hoc analysis showed significant differences between IT and IK mode in torque-angle relationship on the whole range of motion except at $55^{\circ}$ and $90^{\circ}$ at $120 \%$ of $1 \mathrm{RM}$ and at $50^{\circ}$ and $90^{\circ}$ at $100 \%$ of $1 \mathrm{RM}$ (Fig. 2A). The velocity-angle relationship showed significant differences between IT and IK mode from $30^{\circ}$ to $65^{\circ}$ and at $90^{\circ}$ at $120 \%$, and from $30^{\circ}$ to $65^{\circ}$ and from $80^{\circ}$ to $90^{\circ}$ at $100 \%$ (Fig. 2B).

\subsection{Controlled parameters}

Bland-Altman analysis showed concordance between IT and IK modes for the amount of work (Fig. 3A) and for the mean angular velocity (Fig. 3B) as illustrated by the low bias values for the amount of work (6.8 Jat $120 \%$;-2.7 Jat $100 \%$ ) and angularvelocity $\left(-0.9^{\circ} \mathrm{s}^{-1}\right.$ at $120 \% ; 1.8 \% \mathrm{~s}$ at $\left.100 \%\right)$. IT angular velocity variation was equal to $\pm 8.2^{\circ} \mathrm{s}^{-1}$ at $120 \%$ of $1 \mathrm{RM}$ and $\pm 4.2^{\circ} \mathrm{s}^{-1}$ at $100 \%$ of $1 \mathrm{RM}$ across trials. Changing the order between IT and IK sets induced a lower, but acceptable concordance for amount of work (bias =6.8J; CI 95\%: [-59.5-73.0]) and angular velocity (bias $=$ $-0.9^{\circ} \mathrm{s}^{-1}$; CI 95\%: [-7.1-7.4]). ICC and SEM values demonstrated

\section{B}
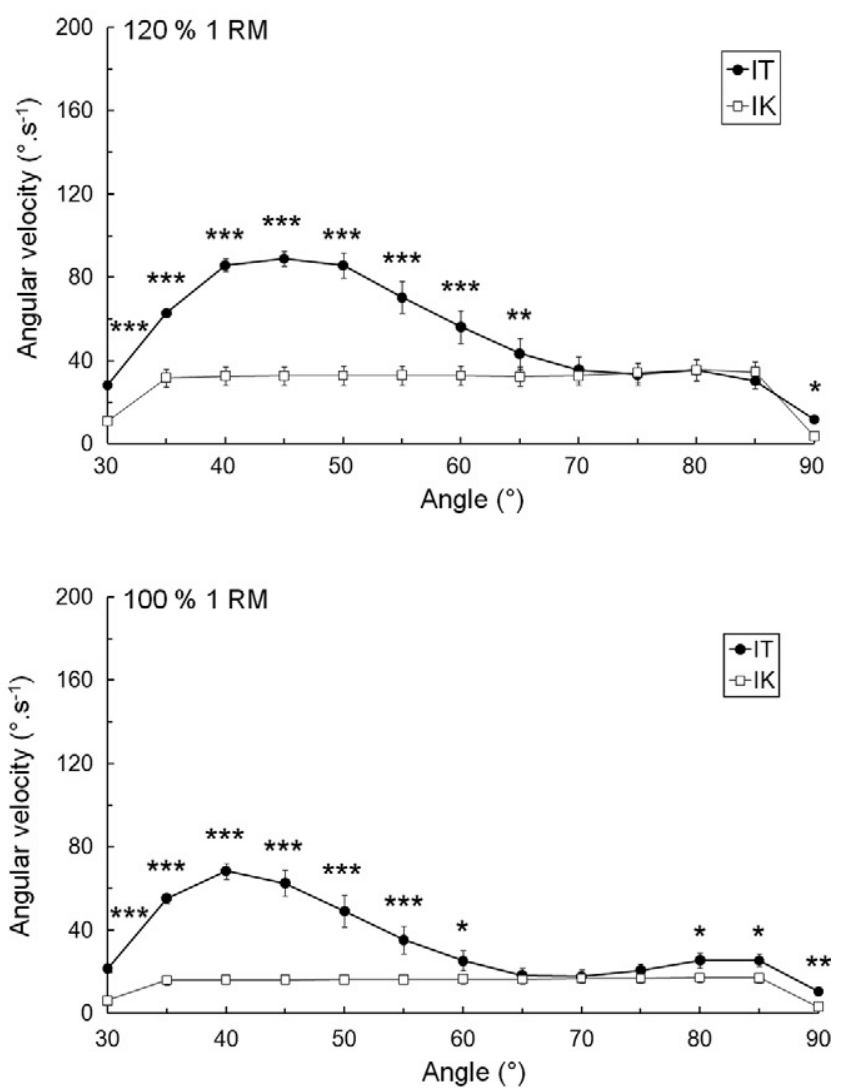

Fig. 2. Muscular torque-angle relationships (A) and angular velocity-angle (B) relationship for isotonic (IT) and isokinetic (IK) eccentric exercises when isotonic mode is performed at $120 \%$ (top) and $100 \%$ (bottom) of the maximal repetition (1RM). All values are means \pm SE. ${ }^{*} p<0.05 ;{ }^{* * *} p<0.01 ;{ }^{* * * *} p<0.001$ (Newman-Keuls $p o s t-h o c$ test). 

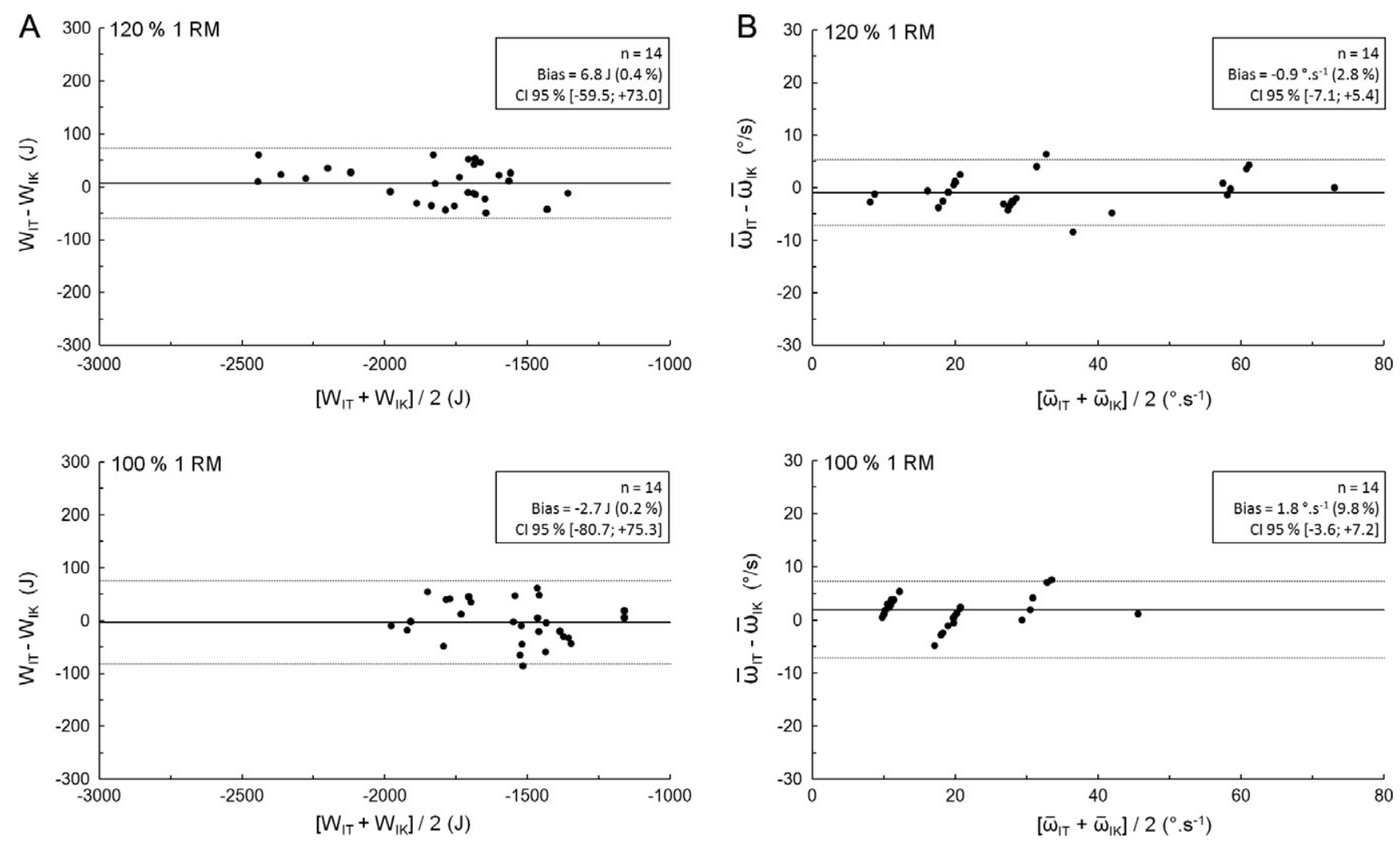

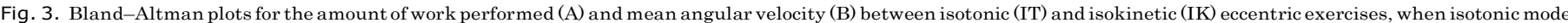

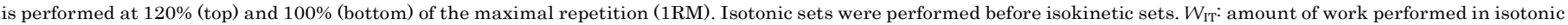
mode; $W_{\mathrm{IK}}$ : amount of work performed in isokinetic mode; $\overline{\mathrm{X}_{\mathrm{IT}}}$ : mean angular velocity in isotonic mode; $\overline{\mathrm{IK}}$ : mean angular velocity in IK mode; CI: confidence interval.

Table 2

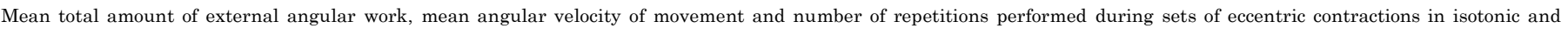
isokinetic modes.

\begin{tabular}{|c|c|c|c|c|c|c|}
\hline \multirow[t]{2}{*}{ Controlled parameter } & \multirow[t]{2}{*}{ Test session } & \multirow[t]{2}{*}{ IT load (\% 1RM) } & \multicolumn{2}{|c|}{ IT mode } & \multicolumn{2}{|c|}{ IK mode } \\
\hline & & & Set 1 & Set 2 & Set 1 & Set 2 \\
\hline \multirow[t]{3}{*}{ Amount of work $(\mathrm{J})$} & 1 & 120 & $\begin{array}{l}-1845 \\
( \pm 87)\end{array}$ & $\begin{array}{l}-1799 \\
( \pm 80)\end{array}$ & $\begin{array}{l}-1857 \\
( \pm 91)\end{array}$ & $\begin{array}{l}-1801 \\
( \pm 83)\end{array}$ \\
\hline & 2 & 120 & $\begin{array}{l}-1860 \\
( \pm 81)\end{array}$ & $\begin{array}{l}-1837 \\
( \pm 80)\end{array}$ & $\begin{array}{l}-1863 \\
( \pm 78)\end{array}$ & $\begin{array}{l}-1777 \\
( \pm 91)\end{array}$ \\
\hline & 3 & 100 & $\begin{array}{l}-1566 \\
( \pm 63)\end{array}$ & $\begin{array}{l}-1567 \\
( \pm 63)\end{array}$ & $\begin{array}{l}-1565 \\
( \pm 67)\end{array}$ & $\begin{array}{l}-1563 \\
( \pm 64)\end{array}$ \\
\hline \multirow[t]{3}{*}{ Angular velocity $\left({ }^{\circ} \mathrm{s}^{-1}\right)$} & 1 & 120 & $\begin{array}{l}28.8 \\
( \pm 4.2)\end{array}$ & $\begin{array}{l}34.7 \\
( \pm 6.0)\end{array}$ & $\begin{array}{l}30.1 \\
( \pm 4.3)\end{array}$ & $\begin{array}{l}35.2 \\
( \pm 5.5)\end{array}$ \\
\hline & 2 & 120 & $\begin{array}{l}32.1 \\
( \pm 4.6)\end{array}$ & $\begin{array}{l}34.4 \\
( \pm 4.6)\end{array}$ & $\begin{array}{l}30.9 \\
( \pm 4.0)\end{array}$ & $\begin{array}{l}35.0 \\
( \pm 5.8)\end{array}$ \\
\hline & 3 & 100 & $\begin{array}{l}20.7 \\
( \pm 3.2)\end{array}$ & $\begin{array}{l}18.7 \\
( \pm 2.3)\end{array}$ & $\begin{array}{l}18.4 \\
( \pm 3.1)\end{array}$ & $\begin{array}{l}17.3 \\
( \pm 2.3)\end{array}$ \\
\hline \multirow[t]{3}{*}{ Number of repetitions } & 1 & 120 & $\begin{array}{l}8.0 \\
( \pm 0.0)\end{array}$ & $\begin{array}{l}8.0 \\
( \pm 0.0)\end{array}$ & $\begin{array}{l}7.7 \\
( \pm 0.2)\end{array}$ & $\begin{array}{l}7.9 \\
( \pm 0.2)\end{array}$ \\
\hline & 2 & 120 & $\begin{array}{l}8.0 \\
( \pm 0.0)\end{array}$ & $\begin{array}{l}8.0 \\
( \pm 0.0)\end{array}$ & $\begin{array}{l}8.1 \\
( \pm 0.2)\end{array}$ & $\begin{array}{l}7.8 \\
( \pm 0.2)\end{array}$ \\
\hline & 3 & 100 & $\begin{array}{l}8.0 \\
( \pm 0.0)\end{array}$ & $\begin{array}{l}8.0 \\
( \pm 0.0)\end{array}$ & $\begin{array}{l}7.3^{*} \\
( \pm 0.3)\end{array}$ & $\begin{array}{l}6.7^{*} \\
( \pm 0.6)\end{array}$ \\
\hline
\end{tabular}

1RM: maximal repetition. TS: test session. Results are presented as mean \pm SE. ${ }^{*} p<0.05$

a satisfying reproducibility for the amount of work $(\mathrm{ICC}=0.96$; $\mathrm{SEM}=75.6 \mathrm{~J} ; \mathrm{CV}=3.0 \%$ )

\subsection{Number of repetitions}

In these standardized conditions, no statistical differences were observed in the number of repetitions performed in IT mode at $120 \%$ of $1 \mathrm{RM}$ and IK mode. When IT sets were performed at $100 \%$ of 1RM, subjects performed more repetitions than in the corresponding IK sets (8.0 and 8.0 in IT vs. 7.3 and 6.7 in IK mode; $p=0.02$ for set $1 ; p=0.005$ for set 2 ; Table 2 ).

\subsection{Performance changes}

Three-way ANOVAs revealed no differences in the amount of work performed ( $p=0.22$ at $120 \% ; p=0.85$ at $100 \%$ ) and the mean 


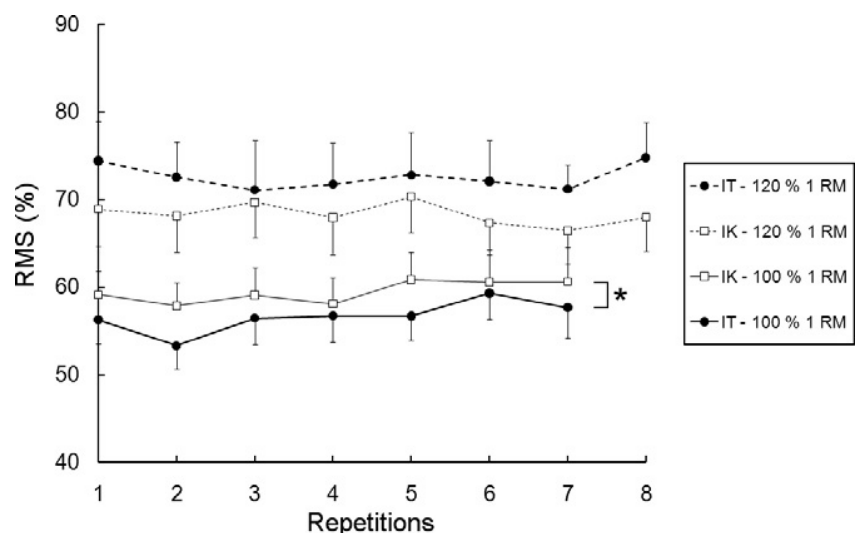

Fig. 4. Root Mean Square (RMS)-repetition relationships for isotonic (IT) and isokinetic (IK) eccentric exercises, when isotonic mode is performed at $100 \%$ and $120 \%$ (dashed line) of the maximal repetition (1RM). All values are means $\pm \mathrm{SE}$. ${ }^{*} p<0.05$.

angular velocity $(p=0.33$ at $120 \% ; p=0.78$ at $100 \%)$ between repetitions for the two solicitation modes. During familiarization session, we observed no differences in peak torque of the two MVCs performed before or after the IT set.

\subsection{EMG activity-repetitions relationships}

The three-way ANOVA on RMS values of EMG activity of quadriceps femoris revealed no set ( $p=0.24$ at $120 \% ; p=0.77$ at $100 \%)$ or repetition effect ( $p=0.86$ at $120 \% ; p=0.86$ at $100 \%$ ). RMS values for the IK mode were higher at $100 \%$ of $1 \mathrm{RM}$ ( $p=0.04$; Fig. 4 ).

\section{Discussion}

The main purpose in the present study was to develop a method to equalize the amount of work and the mean angular velocity in isotonic and isokinetic eccentric exercises. This procedure was designed to be able to compare the effects of IT and IK eccentric exercises on the neuromuscular system during an exercise session and after a training period in future studies. The standardization procedure showed the concordance of amount of work and angular velocity between IT and IK modes. In these standardized conditions, both modes involved the same number of repetitions when IT eccentric exercise was set at $120 \%$ of $1 \mathrm{RM}$. More repetitions were executed in IK mode when IT sets were performed at $100 \%$ of $1 \mathrm{RM}$.

The comparison analysis showed that IT and IK eccentric exercises elicit different mechanical characteristics, including torque and movement velocity (Fig 2). In fact, muscular torque exerted in the IT mode was constant from $35^{\circ}$ to $80^{\circ}$ whereas it increased in IK mode from $30^{\circ}$ to $70^{\circ}$ before decreasing until $90^{\circ}$. Muscular torque was higher in the IT mode at the extended joint angles while it was higher in IK mode at the flexed joint angles. Angular velocity was higher in IT mode in extended and at flexed joint angles at both exercise intensities.

These differences in mechanical load could induce specific effects on neuromuscular system, thus requiring the development of a standardization procedure. Such an approach was successfully undertaken in concentric contractions to equalize external work and movement velocity of IT and IK modes (Remaud et al., 2005). The procedure developed in our study consisted in the next step of this work by implementing the method to eccentric exercise. Previous eccentric protocols using submaximal loads (i.e. $<100 \%$ 1RM) were unable to progressively increase external resistance for optimal eccentric training effects to take place (Hortobagyi and Katch, 1990; Johnson, 1972). Therefore the present study used maximal and supra-maximal loads of $100 \%$ and $120 \%$ of $1 \mathrm{RM}$ that are commonly prescribed in eccentric exercise (Brandenburg and Docherty, 2002; Colson et al., 1999). IT and IK eccentric exercises were standardized by equalizing the amount of work and angular velocity in both modes. Our results showed concordance of the two controlled parameters (i.e. work, angular velocity) between modes, regardless of the order in which they were performed. The standardization procedure also induced a similar duration of eccentric contraction in IT and IK modes. Therefore, it could be assumed that the IT and IK eccentric exercises were standardized for these two parameters. The amount of work performed during eccentric sessions was consistent between each set and no differences existed in maximal isometric peak torque before IT and before IK sets. Consequently, subjects conserved similar capacity of force production in IT and IK sets. These results paralleled the constant activation level of the knee extensors muscles.

In these standardized conditions, the same number of repetitions were necessary to reach the amount of external work performed in the corresponding IT sets at $120 \%$ of $1 \mathrm{RM}$, thus confirming our hypothesis at this exercise intensity. This is in accordance with previous work performed on IT and IK modes at lower intensity with concentric contractions (Remaud et al., 2005). The higher amount of work performed in our study than in the study of Remaud et al. (2005) could be attributed to the higher torque levels the muscles can exert in eccentric contractions compared to concentric contractions. Nevertheless, it is clear that the exercise intensity had an effect on the standardization procedure, since fewer repetitions were needed in IK sets when IT sets were performed at $100 \%$ of $1 \mathrm{RM}$. The muscular torque produced in the IT mode at $100 \%$ were lower than those produced at $120 \%$ of 1RM, resulting in a lower amount of work. Corresponding maximal eccentric contractions in the IK mode produced similar torque levels in all sessions. Consequently, subjects required fewer repetitions in IK mode to achieve the same amount of work as during IT sets performed at $100 \%$ of $1 \mathrm{RM}$. These results are supported by the higher RMS values in IK sets when IT sets were performed at $100 \%$ of 1RM. Indeed, subjects seem to recruit fewer motor units in IT mode in this condition and in turn, produced lower levels of torque.

Initially suggested by Stanish et al. (1986), eccentric exercise is a popular non-invasive treatment for tendinopathy. IT (Jonsson and Alfredson, 2005; Visnes and Bahr, 2007; Young et al., 2005) and IK (Croisier et al., 2007; Stanish et al., 1986) modes have been prescribed to manage this disability that is a major component of clinical injuries in sport and in the general population. Although chances of improvement of knee function and pain are estimated to be $50-70 \%$, comparative studies have to date failed to determine the most effective protocols because of limitations in the study design (Langberg and Kongsgaard, 2008; Meyer et al., 2009; Rees et al., 2009; Visnes and Bahr, 2007). Young et al. (2005) attempted to compare these protocols. However, several parameters were uncontrolled and non-standardized. Moreover, IT and IK eccentric exercises are included in resistance training programs to achieve an increase in muscular strength. However, comparative analysis led in the present study showed significant disparities between both modes in torque and angular velocity-angle relationships. Such differences suggest specific neuromuscular adaptations in response to each mode. Future research is needed to compare the effectiveness of IT and IK modes during eccentric contraction in rehabilitation and training by equalizing work and movement velocity with the present method. Moreover, testing with female and elderly subjects would allow standardization of the procedure on different populations. Indeed the age and gender of participants could have an effect on muscle function and potentially impact this method developed using college-aged male subjects. 


\section{Acknowledgements}

This study was supported by grants from the «Région des Pays de la Loire» and the "Réseau Recherche et Sport en Pays de la Loire» (France). The authors are grateful to the subjects for having accepted to participate in this study and Philippe Sarrazin for his technical assistance. They also thank Ashleigh Kennedy for her input.

\section{References}

Aagaard P, Simonsen EB, Trolle M, Bangsbo J, Klausen K. Isokinetic hamstring/ quadriceps strength ratio: influence from joint angular velocity, gravity correction and contraction mode. Acta Physiol Scand 1995;154(4):421-7.

Abernethy P, Wilson G, Logan P. Strength and power assessment. Issues, controversies and challenges. Sports Med 1995;19(6):401-17.

Babault N, Pousson M, Ballay Y, Van Hoecke J. Activation of human quadriceps femoris during isometric, concentric, and eccentric contractions. J Appl Physiol 2001;91(6):2628-34.

Bland JM, Altman DG. Statistical methods for assessing agreement between two methods of clinical measurement. Lancet 1986;1(8476):307-10.

Blazevich AJ, Cannavan D, Coleman DR, Horne S. Influence of concentric and eccentric resistance training on architectural adaptation in human quadriceps muscles. J Appl Physiol 2007;103(5):1565-75.

Brandenburg JP, Docherty D. The effects of accentuated eccentric loading on strength, muscle hypertrophy, and neural adaptations in trained individuals. $J$ Strength Cond Res 2002;16(1):25-32.

Caruso JF, Hamill JL, Hernandez DA, Yamauchi M. A comparison of isoload and isoinertial leg press training on bone and muscle outcomes. J Strength Cond Res 2005;19(3):592-8.

Colson S, Pousson M, Martin A, Van Hoecke J. Isokinetic elbow flexion and coactivation following eccentric training. J Electromyogr Kinesiol 1999;9(1):1320.

Croisier JL, Foidart-Dessalle M, Tinant F, Crielaard JM, Forthomme B. An isokinetic eccentric programme for the management of chronic lateral epicondylar tendinopathy. Br J Sports Med 2007;41(4):269-75.

Duclay J, Martin A, Robbe A, Pousson M. Spinal reflex plasticity during maximal dynamic contractions after eccentric training. Med Sci Sports Exerc 2008;40(4):722-34.

Guilhem G, Cornu C, Guével A. [Neuromuscular and muscle-tendon unit adaptations to isotonic and isokinetic eccentric exercise]. Ann Readapt Med Phys, in press-a.

Guilhem G, Cornu C, Nordez A, Guével A. A new device to study isoload eccentric exercise. J Strength Cond Res, in press-b.

Hakkinen K, Kallinen M, Komi PV, Kauhanen H. Neuromuscular adaptations during short-term "normal" and reduced training periods in strength athletes. Electromyogr Clin Neurophysiol 1991;31(1):35-42.

Hermens HJ, Freriks B, Disselhorst-Klug C, Rau G. Development of recommendations for SEMG sensors and sensor placement procedures. J Electromyogr Kinesiol 2000;10(5):361-74.

Hortobagyi T, Barrier J, Beard D, Braspennincx J, Koens P, Devita P, et al. Greater initial adaptations to submaximal muscle lengthening than maximal shortening. J Appl Physiol 1996a;81(4):1677-82.

Hortobagyi T, Hill JP, Houmard JA, Fraser DD, Lambert NJ, Israel RG. Adaptive responses to muscle lengthening and shortening in humans. J Appl Physiol 1996b;80(3):765-72.

Hortobagyi T, Katch FI. Role of concentric force in limiting improvement in muscular strength. J Appl Physiol 1990;68(2):650-8.

Housh DJ, Housh TJ, Weir JP, Weir LL, Evetovich TK, Donlin PE. Effects of unilateral eccentric-only dynamic constant external resistance training on quadriceps femoris cross-sectional area. J Strength Cond Res 1998;12(3):192-8.

Johnson BL. Eccentric vs. concentric muscle training for strength development. Med Sci Sports Exerc 1972;4(2):111-5.

Jonsson P, Alfredson H. Superior results with eccentric compared to concentric quadriceps training in patients with jumper's knee: a prospective randomised study. Br J Sports Med 2005;39(11):847-50.

Kingma JJ, de Knikker R, Wittink HM, Takken T. Eccentric overload training in patients with chronic Achilles tendinopathy: a systematic review. $\mathrm{Br}$ J Sports Med 2007;41(6):e3.

Komi PV, Buskirk ER. Effect of eccentric and concentric muscle conditioning on tension and electrical activity of human muscle. Ergonomics 1972;15(4):417-34.

Komi PV, Kaneko M, Aura O. EMG activity of the leg extensor muscles with special reference to mechanical efficiency in concentric and eccentric exercise. Int $\mathrm{J}$ Sports Med 1987;8(Suppl. 1):22-9.

Kovaleski JE, Heitman RH, Trundle TL, Gilley WF. Isotonic preload versus isokinetic knee extension resistance training. Med Sci Sports Exerc 1995;27(6):895-9.

Langberg H, Kongsgaard M. Eccentric training in tendinopathy-more questions than answers. Scand J Med Sci Sports 2008;18(5):541-2.

Linnamo V, Moritani T, Nicol C, Komi PV. Motor unit activation patterns during isometric, concentric and eccentric actions at different force levels. J Electromyogr Kinesiol 2003;13(1):93-101.

Maisetti O, Guevel A, Legros P, Hogrel JY. SEMG power spectrum changes during a sustained 50\% Maximum Voluntary Isometric Torque do not depend upon the prior knowledge of the exercise duration. J Electromyogr Kinesiol 2002;12(2):103-9.

Meyer A, Tumilty S, Baxter GD. Eccentric exercise protocols for chronic non insertional Achilles tendinopathy: how much is enough? Scand J Med Sci Sports 2009;19(5):609-15.

Rees JD, Wolman RL, Wilson A. Eccentric exercises; why do they work, what are the problems and how can we improve them? Br J Sports Med 2009;43(4):242-6.

Remaud A, Cornu C, Guével A. A methodologic approach for the comparison between dynamic contractions: influences on the neuromuscular system. J Athl Train 2005;40(4):281-7.

Remaud A, Cornu C, Guével A. Agonist muscle activity and antagonist muscle coactivity levels during standardized isotonic and isokinetic knee extensions. Electromyogr Kinesiol 2009;19(3):449-58.

Roig M, O'Brien K, Kirk G, Murray R, McKinnon P, Shadgan B, et al. The effects of eccentric versus concentric resistance training on muscle strength and mass in healthy adults: a systematic review with meta-analysis. $\mathrm{Br} \mathrm{J}$ Sports Med 2009;43(8):556-68.

Smith MJ, Melton P. Isokinetic versus isotonic variable-resistance training. Am J Sports Med 1981;9(4):275-9.

Stanish WD, Rubinovich RM, Curwin S. Eccentric exercise in chronic tendinitis. Clin Orthop Relat Res 1986(208):65-8.

Visentini PJ, Khan KM, Cook JL, Kiss ZS, Harcourt PR, Wark JD. The VISA score: an index of severity of symptoms in patients with jumper's knee (patellar tendinosis). Victorian Institute of Sport Tendon Study Group. J Sci Med Sport 1998;1(1):22-8.

Visnes H, Bahr R. The evolution of eccentric training as treatment for patellar tendinopathy (jumper's knee): a critical review of exercise programmes. $\mathrm{Br} \mathrm{J}$ Sports Med 2007;41(4):217-23.

Young MA, Cook JL, Purdam CR, Kiss ZS, Alfredson H. Eccentric decline squat protocol offers superior results at 12 months compared with traditional eccentric protocol for patellar tendinopathy in volleyball players. $\mathrm{Br} \mathrm{J}$ Sports Med 2005;39(2):102-5.

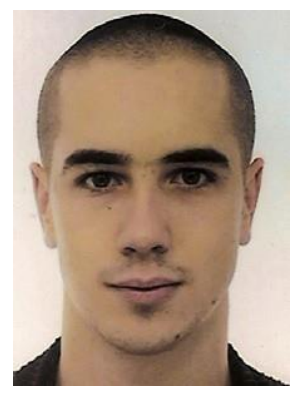

Gaël Guilhem received the Master degree in 2006 in Biology and Physiology from the University of Paris $\mathrm{V}$, and the $\mathrm{PhD}$ degree in 2010 in Sport Sciences from the University of Nantes, France. He is currently researcher at the National Institute for Sports (INSEP) in Paris. His major research interests focus on neu romuscular adaptations to acute and chronic exercise based on surface electromyography, ultrasonography, myostimulation and muscle damages markers.

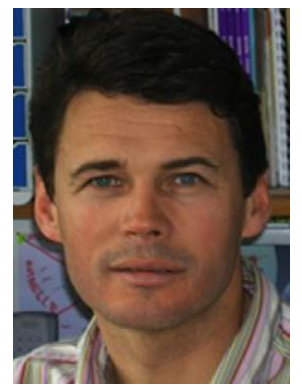

Arnaud Guével received the PhD degree in Sports Sciences from University of Méditerranée, Marseille, France, in 1997. He joined the Faculty of Sports Sciences from the University of Nantes in 1998. His research interests focus on the evaluation of neuromuscular fatigue based on the surface EMG analysis and on the investigation of sports performance and the effects of training on the neuromuscular function.

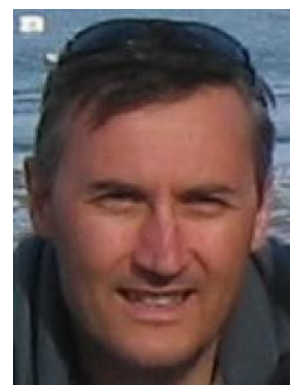

Christophe Cornu received the PhD degree in 1998 in Biomechanics from the University of Technology of Compiègne, France. In 2002, he joined the Depart ment of Sport Sciences at the University of Nantes. His main field of competence is human biomechanics and exercise physiology focusing on the characterization of the musculo-articular complex and on the neuromuscular system plasticity. 